\title{
Laser Light-Scattering Spectroscopy: Preliminary Results on Bioassay of Cystic Fibrosis Factor(s)
}

\author{
PEDRO VERDUGO, THOMAS R. HINDS, AND FRANK F. VINCENZI \\ WITH THE TECHNICAL ASSISTANCE OF LYNN B. LANGLEY AND FRANCES HUHNDORF \\ Center for Bioengineering and Department of Biological Structure (P.V.), and Department of Pharmacology, (T.R.H., \\ F.F.V.), School of Medicine, University of Washington, Seattle, Washington, USA
}

\begin{abstract}
Summary
The effect of 7 cystic fibrosis sera and 4 normal sera was investigated in 37 cultures of ciliated epithelium of the rabbit trachea. Serum was introduced in the Rose culture chambers in a concentration of $10 \%$ by volume. The frequency of ciliary beat was monitored by laser light-scattering spectroscopy. The results show that the response of cilia to sera can be well characterized by laser light scattering. Our preliminary evidence suggests that an early increase in the frequency of ciliary beat, followed by rapid arrest of ciliary activity, is a sensitive and characteristic response produced by cystic fibrosis serum, and not by normal serum.
\end{abstract}

\section{Speculation}

The present work was aimed at demonstrating the feasibility of implementing an objective and quantifiable bioassay for cystic fibrosis factor(s). Laser light-scattering spectroscopy is applicable to detection and quantification of ciliary activity. The method may lead to improved bioassays for cystic fibrosis factor(s).

The observation that serum as well as other biologic fluids from patients with cystic fibrosis (CF) can interfere with ciliary activity has attracted a great deal of interest. Ciliated cells are responsive to so-called CF factor(s) present in serum $(2,5,7,17)$, urine (4), and saliva (9). The establishment of an objective and quantifiable bioassay to detect the presence of ciliotoxic factor(s) would be a useful step toward substantiating the diagnosis of CF on a reliable biochemical or immunochemical basis. A common assumption has been that a quantifiable bioassay for cystic fibrosis, based on the effect of CF serum on ciliary activity, has already been developed. However, visual observation of cilia cannot be considered an objective and quantifiable method even if it has produced excellent results in some laboratories. Investigator bias can be reduced by performing the assay in a "double blind" fashion. Nevertheless, the assay is inherently difficult and subjective partly because one must attempt to visualize objects which are often moving at frequencies greater than the critical flicker fusion frequency.

There are several sources of uncertainty which should be evaluated in order to assess the reliability of ciliary assays: 1) biological variability in the reactivity of the ciliated tissues arising from the source of the tissue, the culturing method, the age of the cells, etc.; 2) variability associated with the serum; i.e., its intrinsic biologic strength, storage procedures, handling methods, etc.; and 3) uncertainties inherent in the assessment of ciliary activity. Without an objective and quantifiable method to measure and record ciliary activity, it is difficult to evaluate the degree of uncertainty introduced by 1 and 2 . Only by developing an objective and quantifiable procedure to record results can standards be set to properly compare the observations of different groups.

Laser light-scattering spectroscopy has recently been applied to measurement of ciliary activity (12). Strict comparison with high- speed cinematography has shown that the laser technique renders precise measurement of the frequency of ciliary beating (13). The present paper reports on preliminary results obtained by laser light-scattering spectroscopy in detecting and recording the effect of $\mathrm{CF}$ and normal sera on the ciliary activity of cultures of ciliated cells of rabbit trachea.

\section{MATERIALS AND METHODS}

\section{SERUM SAMPLES}

Blood was collected from six CF patients attending the Cystic Fibrosis Clinic at the Children's Orthopedic Hospital in Seattle, Washington. The criterion for inclusion was elevated sweat chloride. None of the patients had asthma or other respiratory diseases. Four normal adult subjects were employed. Blood was drawn into plain evacuated blood drawing tubes. The samples were allowed to clot and then stored in plastic tubes at $4^{\circ}$ for $10-14 \mathrm{hr}$. Coagulated blood samples were centrifuged in the cold and sera were then frozen and assayed within 7-10 days after collection. Several assays were also performed on a pool of samples of CF serum that had been frozen and stored in plastic tubes for 6 months.

\section{TISSUE CULTURES}

New Zealand White adult rabbits were stunned and their tracheae rapidly removed. To ensure sterility, the whole trachea was carried through four dishes of sterile Hanks' culture medium (pH 7.3) at room temperature. In the first dish extraneous tissue was trimmed off and the trachea was slit longitudinally. Several small pieces were then sectioned from the trachea and rinsed in the second dish. In the third dish, pieces of mucosa approximately $2 \times 4 \mathrm{~mm}$ were dissected and incubated in $0.15 \%$ hyaluronidase in Hanks' medium for 30-40 min for removal of mucus. In the fourth dish, pieces were cut to a smaller size, $1 \mathrm{~mm}$ or less, in fresh Hanks' medium and placed in groups of six on the plastic bottom cover slip of a Rose chamber. The explants were covered with an $8-\mu \mathrm{m}$ pore polycarbonate sieve, which had been previously sterilized and conditioned in tissue culture medium. The culture chambers were filled with $2 \mathrm{ml}$ Eagle's medium (Steinberger modification) with $20 \%$ horse serum ( $\mathrm{pH} 7.35$ ) and incubated at $37^{\circ}$. After 4-5 days the outgrowths of new ciliated cells that developed from the original explants have well synchromized ciliary motion and can be used for the assays described here. Details of the culturing technique have been published elsewhere (16).

\section{DETECTION OF CILIARY ACTIVITY BY LASER LIGHT-SCATTERING SPECTROSCOPY}

Laser light-scattering spectroscopy is based on analysis of the spectrum of the intensity fluctuations of laser light reflected from moving objects. This method has been applied in the study of 
diffusional motion of macromolecules (10), sperm motility (1), and bacterial movement (15). The technique can also be applied to assess ciliary motion $(12,13)$, thereby providing an objective and quantifiable basis for characterizing ciliary activity.

A safe low-power source of laser light should be used. When the laser beam is directed at a ciliated surface, the backscattered light reflected from the moving cilia exhibits fluctuations in intensity. This is due to both the precise frequency and coherency of the laser light and the movement of the reflecting cilia. The fluctuations of the backscattered light can be detected with a photomultiplier tube and the spectral structure of the resulting photocurrents can be assessed either by standard spectrum analysis or autocorrelation techniques. The autocorrelation function of the scattered light fluctuations produced by the periodic movement of a population of cilia is a damped cosine wave. The period of this wave is dependent upon the mean frequency of beating in the population of cilia and its damping rate is related to the distribution of frequencies of ciliary beat in the population (13). The distribution of frequencies and the mean frequency of ciliary beat can also be assessed by spectrum analysis of the intensity fluctuations of the laser-scattered light. Spectrum analysis renders, in the frequency domain, the same information that the autocorrelation function renders in the time domain. Either one can be determined from the other by Fourier transformation.

In our experiments the cultures were first equilibrated in Hanks' solution, pH. $7.3,37^{\circ}$, for $30 \mathrm{~min}$. The Rose chamber was then positioned on a Reichert inverted microscope modified for laserscattering spectroscopy (Fig. 1). The attenuated beam of a He-Ne gas laser (Spectra Physics 124A) was focused on the cultured cells on an area approximately $5000 \mu \mathrm{m}^{2}$, illuminating a population of about 2000 to 4000 cilia, depending on the density of ciliated cells. The scattered light was collected at an angle of $35^{\circ}$ by the lens aperture system of the microscope and focused onto a pinhole in front of the cathode of a photomultiplier tube (RCA-C7164R). The size of the pinhole was about one coherent area in order to secure spatial correlation of the detected scattered light; thereby obtaining a better signal-to-noise ratio. Photocurrent fluctuations were analyzed by an autocorrelator (Saicor-Honeywell Model $43 \mathrm{~A}$ ) or by a Fourier fast transform digital spectrum analyzer (Nicolet Model 440A). A detailed description of the method can be found in the work of Lee and Verdugo (13).

Spontaneous variations in the frequency of ciliary beating are commonly observed in epithelial cilia (11). Such variations were also found in our cultures. Clusters of ciliated cells to be used for serum assay were selected for stability of both the distribution of frequencies and the mean frequency of ciliary beat, since the more unstable these parameters the more difficult is the interpretation of the results (Fig. 2). Groups of cells with incoherent modes of ciliary beat (as shown by a broad frequency distribution in the spectrogram) were not considered useful for this assay. Clusters of cilia which were unstable in the mean frequency of ciliary beat were also not considered useful (even if the distribution of frequency was narrow). Once a group of cells with reasonably stable performance for at least $20-30 \mathrm{~min}$ was found, $200 \mu \mathrm{l}$ serum were injected into the culture chamber. Since our culture chamber contained $2 \mathrm{ml}$, the final volume ratio of serum to Hanks' solution was $1: 10$ (10\% by volume).

\section{RESULTS}

Figure 2 shows two sets of spectrograms obtained by laser lightscattering spectroscopy. This figure illustrates the time course of typical effects of normal and CF serum on the frequency of ciliary beating in cultures of rabbit trachea. Over $35 \mathrm{~min}$, normal serum produced some broadening of the frequency spectrum but only a slight decrease in the mean frequency. By contrast, CF serum produced an early $(4.5 \mathrm{~min})$ increase in ciliary frequency followed by a decrease ( $12 \mathrm{~min})$ and ciliary arrest.

Results obtained from 37 assays performed on 11 different serum samples show that both CF and normal serum may affect and eventually arrest ciliary activity (Table 1). We observed a marked initial increase in ciliary beat frequency in $80 \%$ of the assays with CF sera. An increase but without a susequent decrease in ciliary frequency was produced in only one assay of a normal serum $(8 \%)$, and in four assays of CF sera (16\%). More often, CF sera resulted in a pattern of acceleration followed by rapid arrest of ciliary activity (e.g., Fig. 2). This pattern was observed in $64 \%$ of the assays of CF serum, but was not observed in any of the assays of normal serum. The only case in which this pattern was not recorded with reputed CF serum (CF-0270503, Table 1) was in five assays performed on the serum of a 23-year-old subject, who had no abnormality but a positive sweat chloride test. Normal serum produced arrest of ciliary activity within the first $35 \mathrm{~min}$ in 2 of 12 assays $(16.6 \%)$. In nine others, ciliary activity either decreased slightly or continued at control rates for more than $1 \mathrm{hr}$ after injection of the normal serum (75\%).

No systematic difference in the frequency distribution of ciliary beat was apparent in response to sera. Thus, both normal and $\mathrm{CF}$ serum can produce frequency incoherence. As shown in Figure 2, the range of the frequency distribution can increase transiently under the influence of either normal or CF serum, and secondary peaks can become apparent in the spectrum.

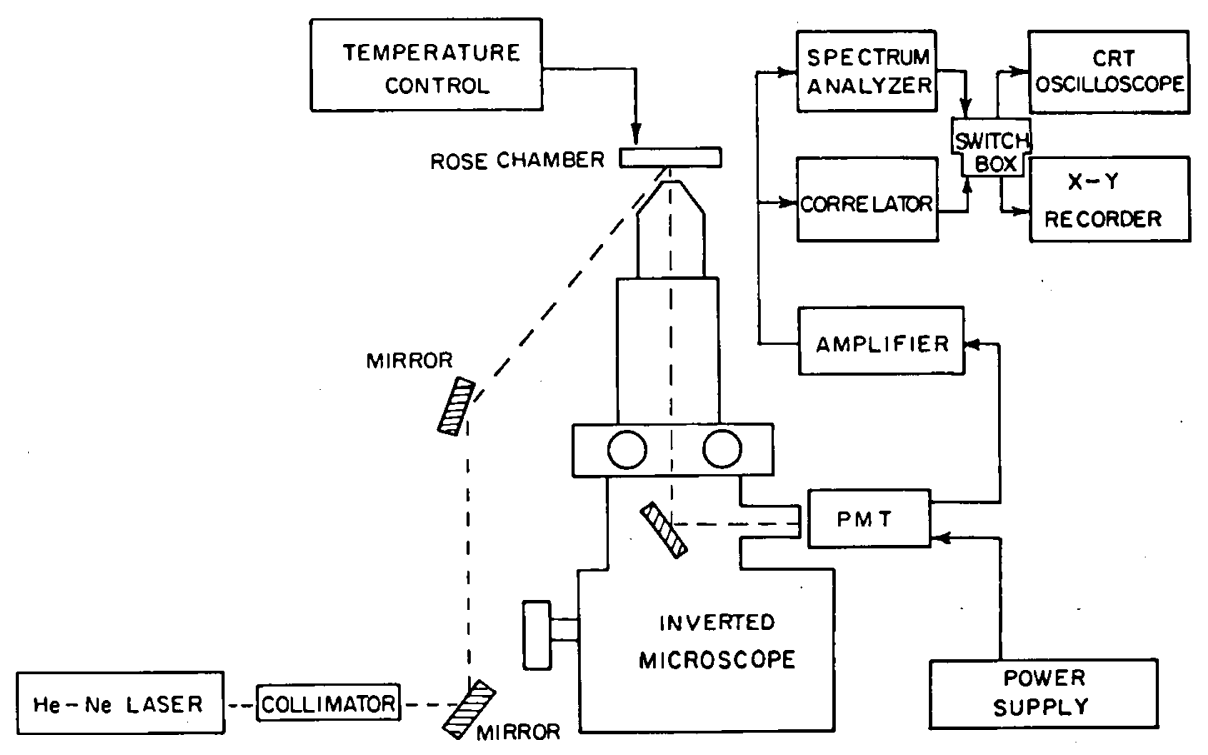

Fig. 1. Schematic diagram of the laser light-scattering spectrometer. 

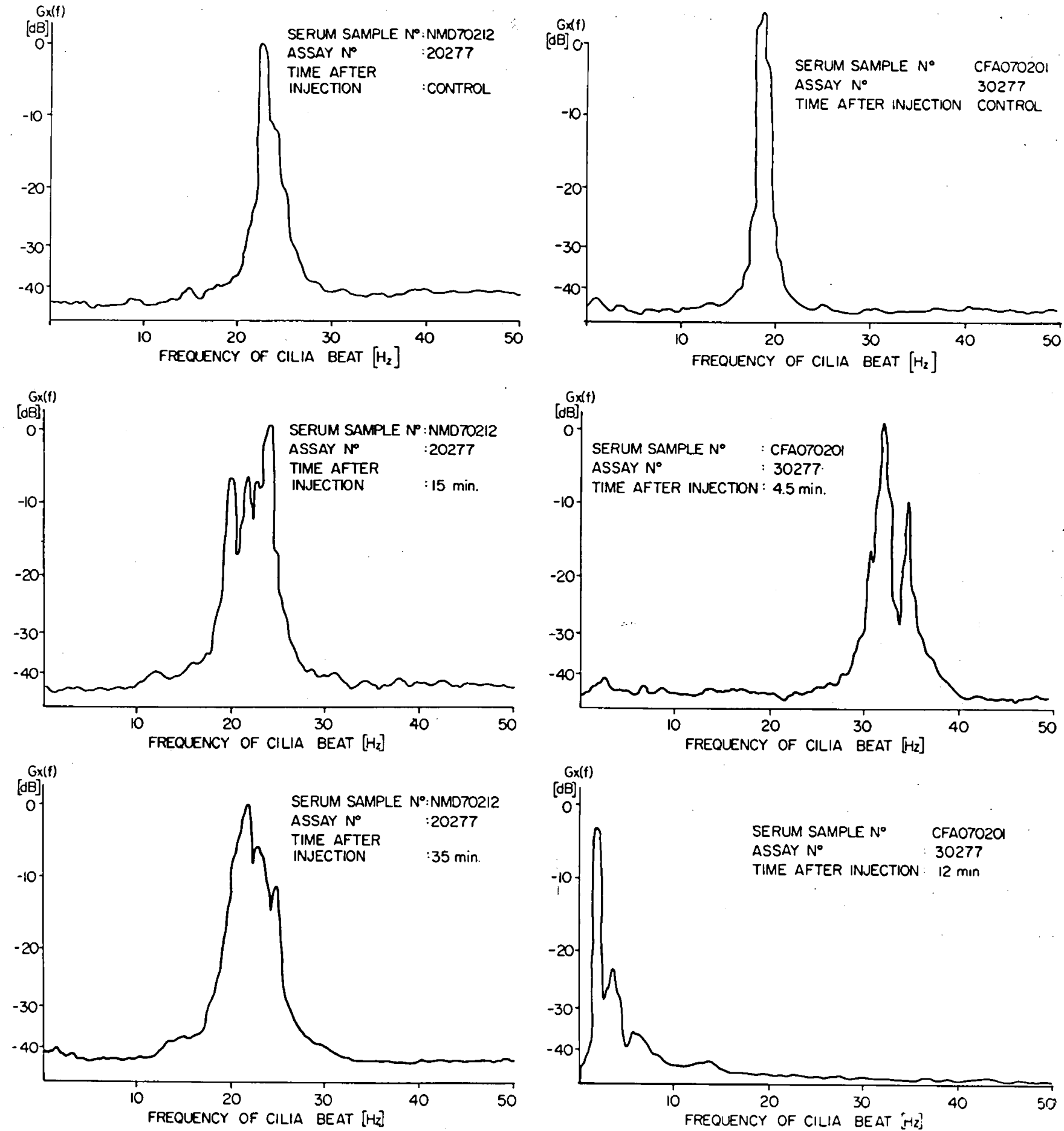

Fig. 2. Effect of normal (left column) and CF (right column) serum on the frequency of ciliary beat in cultures of rabbit trachea as measured by laser light-scattering spectroscopy. The spectrograms show the relative distribution of spectral components [power spectral density $\mathrm{G} \times(\mathrm{f})$ ] in decibels $(\mathrm{dB})$ as a function of the frequency of ciliary beat. A random distribution of frequency of ciliary beat is characterized by a flat distribution in the spectrogram. On the contrary, when there is a dominant mode of frequency of ciliary beat the spectrogram shows a peak with a certain distribution around a mean frequency. The more coherent the frequency of ciliary beat the narrower this distribution. The time after injection of serum into the culture chamber is indicated in each spectrogram. Note the marked increase followed by a rapid decrease of the frequency of ciliary beat induced by CF serum.

Although we have not quantified the observation, it was noted that newly formed outgrowths of ciliated cells seem to be more responsive to $\mathrm{CF}$ serum than the original explants of ciliated cells. Under exposure to CF serum, while most of the original explants were still beating at control rate, many groups of younger cells have completed a cycle of acceleration and ciliary arrest. On the other hand, it appears that the younger the cells, the more unstable the frequency and coherency of their ciliary beat. Thus, care should be taken in selecting clusters of stable as well as responsive cells.

\section{DISCUSSION}

The beat frequency of a collection of cilia, as measured by laser light-scattering spectroscopy, can be characterized by a mean value and a certain distribution around the mean. We have 
Table 1. Influence of $C F$ and normal sera on rabbit tracheal ciliary activity ${ }^{1}$

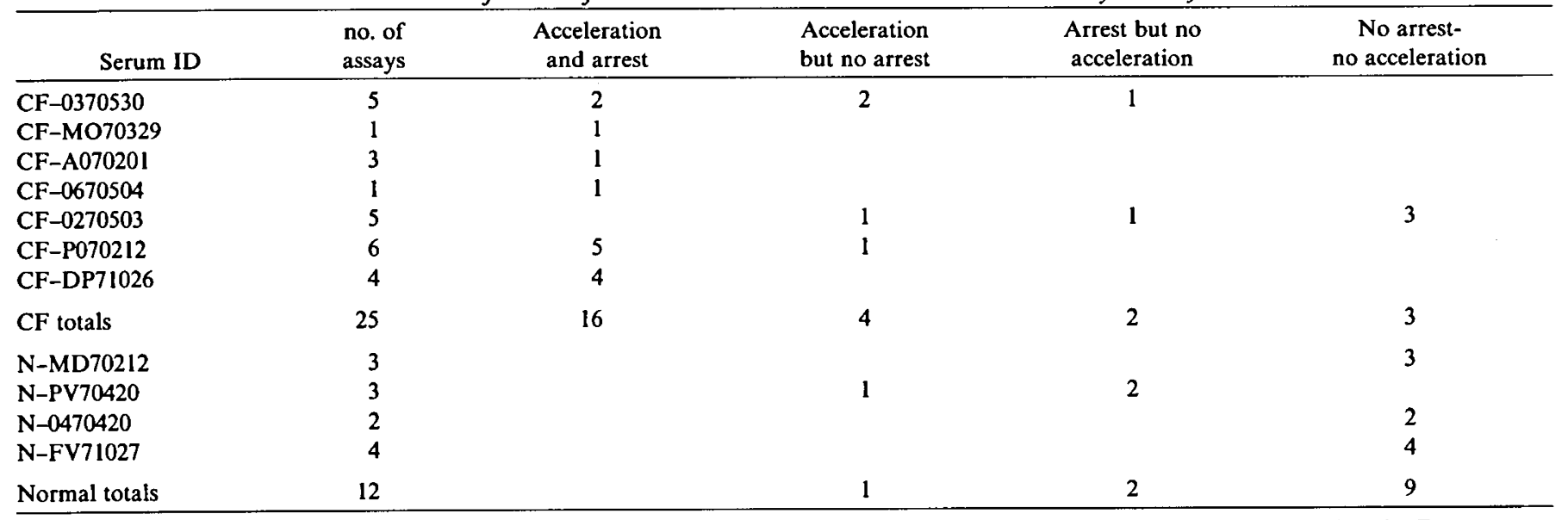

1 The effect of CF and of normal sera on ciliary activity in cultures of ciliated epithelia of the rabbit trachea was monitored for 35 min. Four patterns of reaction were tabulated depending on the presence or absence of an early increase of the frequency of ciliary beat, and on whether or not arrest of ciliary activity was observed.

investigated the effect of a small number of normal and CF sera upon these two parameters of ciliary activity. Thus, this report is one of the first attempts to provide an objective basis for a biologic test that, since its introduction by Spock et al. in 1967 (17), has been based on visual observation.

Although the definition of dyskinesia as a "disorganized ciliary beat" may have a specific meaning for a visual pattern of ciliary beat, it cannot be defined in a physical parametric form. Indeed, both frequency and phase incoherencies can produce visual patterns of "dyskinetic" ciliary activity. Physically, however, these two parameters are quite different. Laser light scattering does not yield information regarding phase coherency. For this reason, our measurements of mean frequency and frequency distribution of ciliary beat do not have a strict counterpart in what has been reported as "dyskinesia."

Our preliminary evidence suggests that inhibition and cessation of ciliary activity is a sensitive but nonspecific criterion with which to identify CF serum. On the other hand, a less sensitive but more characteristic response pattern produced by CF serum (and not by normal serum) is early acceleration followed by rapid arrest of ciliary beat. This is in good agreement with the observations of Wilson et al. (19). We have not yet examined sera of CF carriers. Such assays would help to clarify the possible specificity and usefulness of the technique for identification of $\mathrm{CF}$ and its carrier state.

High-speed cinematography has recently been used to evaluate the effect of CF serum on ciliary motion (6). Cinematography is a very precise method to characterize ciliary activity. In fact, this method was used as a standard for establishing the validity of laser light-scattering spectroscopy $(12,13)$. However, our results do not agree with those obtained by Cheung and Jahn (6) using cinematography. Neither do our results agree with their suggestion that $C F$ sera have no effect on the beat pattern of the respiratory cilia. Differences in handling the sera or the tracheal explants could perhaps account for these different results. Evaluation of the effect of CF sera on ciliary activity is complex and more work is needed.

Recent observations indicate that EGTA can abolish the dyskinetic reaction induced by $C F$ serum. Moreover, normal serum containing the calcium ionophore, A23187, can mimic the effect of CF serum (3). We recently observed that when the ionophore A23187 is injected into a tracheal culture, a pattern characterized by a rapid increase in the frequency of ciliary beat followed by ciliary arrest was observed (18). Such observations may be interpreted to mean that CF factor(s) specifically or nonspecifically short-circuit the cell membrane to $\mathrm{Ca}^{2+}(3,8)$. The role of calcium in the regulation of ciliary activity in mammalians is being eluci- dated $(14,18)$. The present data, as well as our recent observations (18), are compatible with but do not prove the interpretation put forth by Conover et al. (8) and Bogart et al. (3) to explain the mechanism of action of CF factor(s).

The value of the present work does not lie in the weight of the reported data, since they are strictly preliminary. The results appear to demonstrate the feasibility of an objective and quantifiable method to evaluate the effect of CF serum on ciliary activity. The laser light-scattering technique is one of the simplest, most accurate, and reproducible methods for measuring the frequency of ciliary beat. We suggest that this technique should be considered as a possible basis for an objective and quantifiable bioassay for CF factor(s).

\section{REFERENCES AND NOTES}

1. Bérge, P., Volochine, B., Billard, R., and Hamelin, A.: Mise en évidence du mouvement propre de microorganisms vivant grâce a l'étude de la diffusion inélastique de la lumière. C.R. Acad. Sci. D, 256: 889 (1967).

2. Besley, G. T. N., Patrack, A. D., and Northan, A. P.: Inhibition of motility of gill cilia of Dreissensia by plasma of cystic fibrosis patients and their parents. J. Med. Genet., 6: 278 (1969).

3. Bogart, B. I., Conod, E. J., and Conover, J. H.: The biologic activity of cystic fibrosis serum. I. The effect of cystic fibrosis sera and calcium ionophore A23187 on rabbit tracheal explants. Pediat. Res., 11: 131 (1977).

4. Bowman, B. H.: Factors related to cystic fibrosis. In: J. A. Mangos and R. C. Talamo: Cystic Fibrosis, Projections into the Future, pp. 227-248 (Symposia Specialists, NY, 1976).

5. Bowman, B. H., Lockhart, L. H., and McCombs, M. L.: Oyster ciliary inhibition by cystic fibrosis factor. Science, 164: 325 (1969).

6. Cheung, A. T. W., and Jahn, T. L.: High speed cinemicrographic studies on rabbit tracheal (ciliated) epithelia: Cytolytic effects of cystic fibrosis serum on tracheal epithelial cells. Pediatr. Res., 10: 144 (1976).

7. Conover, J. H., Bomforte, R. J., Hathaway, P., Paciuc, S., Conod, E. J., Hirschhorn, K., and Kopel, F. B.: Studies on ciliary dyskinesia factor in cystic fibrosis. I. Bioassay and heterozygote detection in serum. Pediatr. Res., 7: 220 (1973).

8. Conover, J. H., Conod, E. J., Gaerlan, P. F., and Bogart, B. I.: Calcium flux and cystic fibrosis. Lancet, $1: 1362$ (1976).

9. Doggett, R. G., and Harrison, G. M.: Cystic fibrosis: In vitro reversal of the ciliostatic character of serum and parotid secretions by heparin. Nature (Lond) 243: 250 (1973).

10. Dubin, S. B., Lunacek, J. H., and Benedek, G. B.: Observation of the spectrum of light scattered by solutions by biological macromolecules. Proc. Natl. Acad. Sci. USA, 57: 1164 (1967).

11. Eckert, R., and Murakami, A.: Calcium dependence of ciliary activity in the oviduct of the salamander Necturus. J. Physiol (Lond), 226: 699 (1972).

12. Lee, W. I., and Verdugo, P.: Laser light-scattering spectroscopy: a new application in the study of ciliary activity. Biophys. J., 16: 1109 (1976).

13. Lee, W. I., and Verdugo, P.: Ciliary activity by laser light scattering spectroscopy. Ann. Biomed. Eng., 5: 248 (1977).

14. Lee, W. I., Verdugo, P., Schurr, J. M., and Blandau, R. J.: Control of oviductal ciliary activity. Effect of extracellular calcium concentration. Biophys J., I6: 120 (1976).

15. Nossal, R., and Chen, S. H.: Light scattering from motile bacteria. J. Physique Coll. Cl., 33: 171 (1972).

16. Rumery, R. E., Phinney, E., and Blandau, R. J.: Culture of mammalian embry- 
onic ovaries and oviducts. In: J. C. Daniel, Jr.: Methods in Mammalian Embryology, pp. 472-495 (W. H. Freeman and Company, San Francisco, 1970).

17. Spock, A., Heick, H. M., Cress, H., and Logan, W. S.: Abnormal serum factor in patients with cystic fibrosis of the pancreas. Pediatr. Res., 1: 173 (1967).

18. Verdugo, P., Rumery, R. E., and Lee, W. I.: Calcium-induced activation of ciliary activity in mammalian ciliated cells. J. Cell Biol., 75: 293 (1977).

19. Wilson, G. B., Monsher, M. T., and Fudenberg, H. H.: Studies on cystic fibrosis

Copyright (C) 1979 International Pediatric Research Foundation, Inc. $0031-3998 / 79 / 1302-0131 \$ 02.00 / 0$ using isoelectric focusing. III. Correlation between cystic fibrosis protein and ciliary dyskinesia activity in serum shown by a.modified rabbit tracheal bioassay. Pediatr. Res., 11: 143 (1977).

20. This work was partially supported by Grant HL-20191-02 from NIHLB and by a grant from the Cystic Fibrosis Foundation.

21. Received for publication January 6, 1978.

22. Accepted for publication March 29, 1978. 